\title{
Challenges of Measuring Costs of Care for US Practices
}

\author{
Lena M. Chen, MD, MS ${ }^{\top}$ (i), Lok Wong Samson, PhD², Rachael B. Zuckerman, $P h D^{2}$, \\ Karen E. Joynt Maddox, MD, MPH ${ }^{3}$, E. John Orav, PhD',4, and Arnold M. Epstein, MD, \\ $M A^{4,5}$
}

\begin{abstract}
'Division of General Medicine, Department of Internal Medicine, University of Michigan, Ann Arbor, MI, USA; ${ }^{2}$ United States Department of Health and Human Services, Office of the Assistant Secretary for Planning and Evaluation, Washington, DC, USA; ${ }^{3}$ Cardiovascular Division, Department of Medicine, Washington University School of Medicine, St. Louis, MO, USA; ${ }^{4}$ Department of Medicine, Brigham and Women's Hospital, Boston, MA, USA; ${ }^{5}$ Department of Health Policy and Management, Harvard T.H. Chan School of Public Health, Boston, MA, USA.
\end{abstract}

J Gen Intern Med 35(4):1320-2

DOI: $10.1007 / \mathrm{s} 11606-019-05233-\mathrm{x}$

(c) Society of General Internal Medicine 2020

\section{INTRODUCTION}

As the US healthcare system transitions from fee-for-service to value-based purchasing, evaluating physician practice performance on cost is central to driving value. Two measures-Total per Capita Cost (TPCC) and Medicare Spending per Beneficiary (MSPB) - comprise the cost domain in Medicare's mandatory physician value-based purchasing program: the Merit-based Incentive Payment System (MIPS). Therefore, we sought to describe the feasibility of applying claims-based cost measures included in MIPS to practices nationwide, and to assess whether feasibility varies by practice size or specialty mix.

\section{METHODS}

We used national Physician Value-based Payment Modifier (VM) program data collected for payments in 2015 , reflecting performance in 2013. We started with 253,515 practices with Medicare Part B claims. Following the Centers for Medicare \& Medicaid Services' (CMS) criteria for 2018 MIPS participation, we excluded practices with $<200$ Medicare Part B beneficiaries or $<\$ 90,000$ in Part B charges, and practices in the Medicare Shared Savings Program and the Comprehensive Primary Care Initiative, leaving 85,865 MIPS-eligible practices. $^{1}$

Patients were attributed to practices using MIPS specifications: for TPCC, beneficiaries are attributed to the practice (TIN or tax identification number) providing the plurality of primary care services. ${ }^{1}$ For MSPB, beneficiaries are attributed to the TIN with the plurality of Part B billings during an inpatient episode. ${ }^{1}$ TPCC was calculated by summing all Part

${ }^{1}$ Each of the included practices had contact with at least 200 Medicare beneficiaries, but not all of these beneficiaries were attributed to one of the included practices (based on plurality of Evaluation and Management claims), and used for TPCC or MSPB cost reporting.

Published online February 19, 2020
A and B claims for a year; MSPB sums all Part A and B claims three days before hospital admission through 30 days postdischarge (MSPB).

Our primary predictor was practice size as defined by MIPS: small (1-15 eligible professionals or EPs), medium (16-99 EPs), and large (100+ EPs). We categorized practices as primary care provider (PCP) only, PCP plus specialist, or specialist only. PCPs include general practice, pediatrics,

Table 1 Practice, Physician, and Patient Characteristics Stratified by Practice Size

\begin{tabular}{|c|c|c|c|}
\hline Characteristics & $\begin{array}{l}\text { Small } \\
\text { (1-15 EPs) }\end{array}$ & $\begin{array}{l}\text { Medium } \\
\text { (16-99 EPs) }\end{array}$ & $\begin{array}{l}\text { Large } \\
(100+\text { EPs })\end{array}$ \\
\hline$N$, practices & $N=76,481$ & $N=8290$ & $N=1094$ \\
\hline$N$, clinicians & $n=226,055$ & $n=287,281$ & $n=320,098$ \\
\hline \multicolumn{4}{|l|}{ Practice } \\
\hline $\begin{array}{l}\text { Physicians, mean } \\
\text { (median) }\end{array}$ & $2(1)$ & $23(19)$ & $211(121)$ \\
\hline $\begin{array}{l}\text { Clinicians, mean } \\
\text { (median) }\end{array}$ & $3(2)$ & $35(28)$ & $293(173)$ \\
\hline \multicolumn{4}{|l|}{ Practice mix, \% } \\
\hline PCP-only & $25 \%$ & $3 \%$ & $<1 \%$ \\
\hline PCP-specialist & $6 \%$ & $49 \%$ & $86 \%$ \\
\hline Specialist-only & $65 \%$ & $43 \%$ & $11 \%$ \\
\hline No physicians & $4 \%$ & $5 \%$ & $4 \%$ \\
\hline Rural, \% & $16 \%$ & $19 \%$ & $10 \%$ \\
\hline \multicolumn{4}{|l|}{ Clinicians } \\
\hline Age, years, mean & 46 & 47 & 50 \\
\hline Met MU stage $1, \%$ & $30 \%$ & $19 \%$ & $23 \%$ \\
\hline Female, \% & $32 \%$ & $40 \%$ & $45 \%$ \\
\hline \multicolumn{4}{|l|}{ Patient } \\
\hline Age, mean & 73 & 72 & 72 \\
\hline Dually eligible, $\%$ & $19 \%$ & $17 \%$ & $17 \%$ \\
\hline Disabled, $\%$ & $22 \%$ & $23 \%$ & $24 \%$ \\
\hline Medically complex, \% & $22 \%$ & $10 \%$ & $5 \%$ \\
\hline Average HCC score & 1.27 & 1.21 & 1.19 \\
\hline
\end{tabular}

$N$ number of practices, $n$ number of clinicians, EP eligible professional, PCP primary care provider, MU meaningful use, HCC hierarchical condition category

A practice is defined as rural if least three quarters of a practice's providers bill from rural zip codes used by the Federal Office of Rural Health Policy (FORHP). A practice is defined as medically complex if it has an average HCC risk score that was in the top quartile of the HCC risk score for all Medicare beneficiaries. Clinician characteristics were weighted by clinicians and patient characteristics were weighted by attributed beneficiaries. The VM Program (similar to MIPS) attributes fee-for-service Medicare beneficiaries to practices based on where individuals receive the plurality of their primary care services. All comparisons between small and large practices and between medium and large practices were statistically significant at the $p<0.05$ level, except for dual eligible patients in medium or small vs. large practices. Primary care includes general practice, pediatrics, geriatric medicine, internal medicine, or family practice 
a

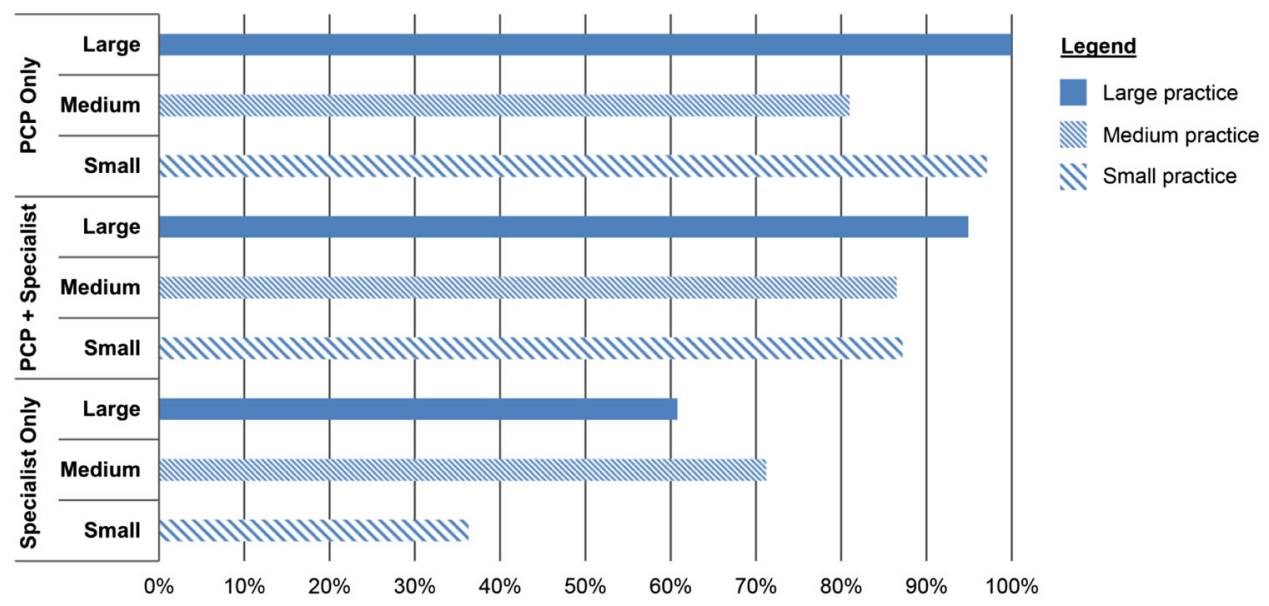

b
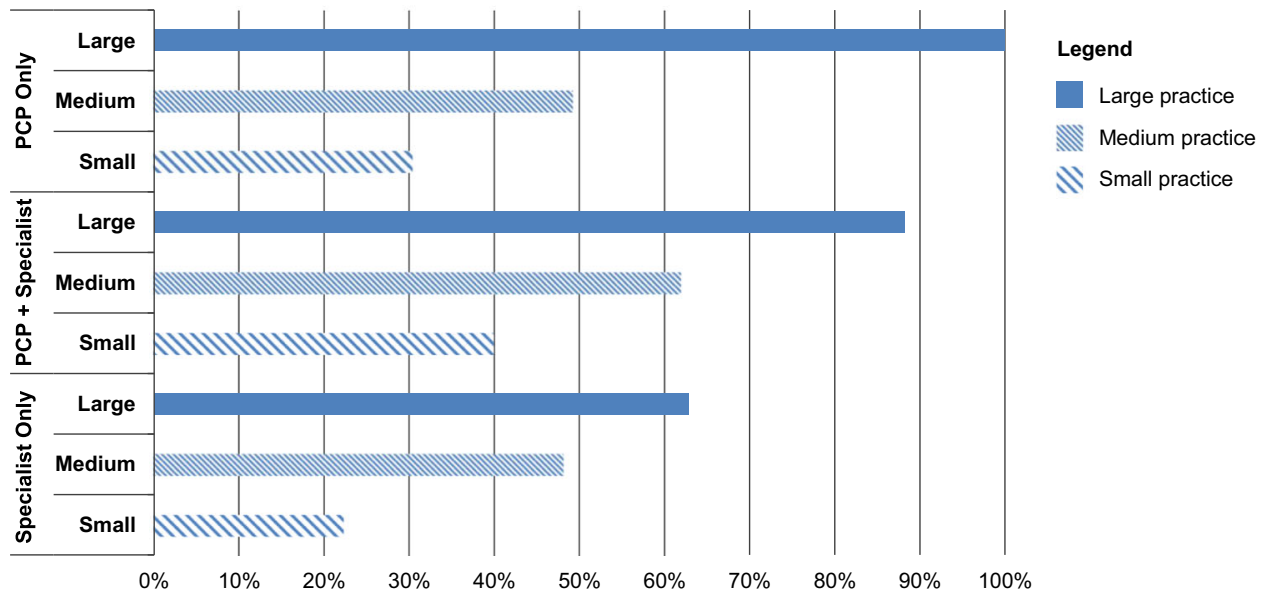

Figure 1 a Proportion of practices meeting minimum case requirements for the Total per Capita Cost (TPCC) measure, stratified by practice size and mix. b Proportion of practices meeting minimum case requirements for Medicare Spending per Beneficiary (MSPB) measure, stratified by practice size and provider mix. Note: PCP is primary care provider. Primary care includes general practice, pediatrics, geriatric medicine, internal medicine, or family practice.

geriatrics, internal medicine, or family practice. Our primary outcome was measure reportability, defined as meeting minimum reporting requirements (20 and 35 attributed beneficiaries for TPCC and MSPB, respectively). The denominator for reportability was practices with at least one qualifying case.

We used descriptive statistics to compare practice characteristics, and to estimate the proportion of practices meeting minimum reporting requirements.

\section{RESULTS}

The study cohort included 76,481 small practices $(89.0 \%$ of practices; $27 \%$ of clinicians), 8290 medium practices (9.7\% of practices; $35 \%$ of clinicians), and 1094 large practices (1.3\% of practices; $38 \%$ of clinicians) (Table 1). Nearly two-thirds of small practices were comprised solely of specialists. For TPCC, practices with at least one PCP usually met case minimums, regardless of practice size (Fig. 1a, top two panels). Fewer specialist-only practices met case minimums (Fig. 1a, bottom panel). For MSPB, large practices were more likely than smaller practices to meet case minimums, regardless of provider mix (Fig. 1b, all panels). Small specialist-only practices were least likely to meet case minimums (Fig. 1b, bottom panel).

\section{DISCUSSION}

Most practices that qualify for MIPS are small, specialist-only practices that are unable to meet minimum case requirements for MIPS' two required cost measures, due to a combination of size and provider mix. This suggests that clinicians in small, specialist-only practices are less likely to be evaluated on cost.

Our study is the first to identify the proportion and types of MIPS-eligible practices that cannot meet case minimums for cost measures. It has implications as policymakers consider options to strengthen MIPS. This year CMS 
introduced 8 episode-based cost measures that providers can elect to link to payment in $2021 .^{2}$ The Medicare Payment Advisory Commission has proposed that physicians band together in larger entities to increase sample size. ${ }^{3}$ Even so, given the narrow clinical scope of episodebased bundles, and the many operational hurdles that face practices that seek to form a virtual group, it is important to identify alternative approaches to ensure that value (i.e., both quality and cost) is rewarded.

Financial Disclosures: This study was funded by the US Department of Health \& Human Services. Lena Chen was supported by funding from AHRQ (Grant No. RO1 HSO24698). At the time this research was conducted, Drs. Chen and Joynt Maddox were affiliated with the Department of Health and Human Services.

Corresponding Author: Lok Wong Samson, PhD; United States Department of Health and Human Services, Office of the Assistant Secretary for Planning and Evaluation, Washington, DC, USA (e-mail: lokwong.samson@hhs.gov).

Author Contributions Drs. Chen and Samson had full access to all of the data in the study and take responsibility for the integrity of the data and the accuracy of the data analysis.

Study concept and design: Chen, Samson, Epstein

Acquisition of data: Chen, Samson, Zuckerman

Drafting of the manuscript: Chen

Revision of the manuscript for important intellectual content: Chen,

Samson, Zuckerman, Joynt Maddox, Orav, Epstein

Statistical analysis: Chen, Samson, Orav

\section{Compliance with ethical standards:}

Conflict of interest: The authors declare that they do not have a conflict of interest.

\section{REFERENCES}

1. 2018 Merit-based Incentive Payment System (MIPS) Cost Performance Category Fact Sheet. https://www.cms.gov/Medicare/Quality-PaymentProgram/Resource-Library/2018Cost-Performance-Category-Fact-Sheet. pdf. Accessed 12 Nov 2018. Of note, the VM Program measured and rewarded performance of practices, so cost measures such as TPCC and MSPB were based on beneficiaries attributed to TINs. In MIPS, beneficiaries are attributed to unique TIN-NPIs (Tax Identification Number-National Provider Identifiers) and performance can be measured at either the TINNPI or TIN level.

2. US Department of Health and Human Services, Centers for Medicare \& Medicaid Services. Medicare Program; Revisions to Payment Policies under the Physician Fee Schedule and Other Revisions to Part B for CY 2019, etc. Document No. 2018-24170. 2018. https://www.federalregister.gov/documents/2018/11/23/2018-24170/medicare-programrevisions-to-payment-policies-under-the-physician-fee-schedule-and-other-revisions. Accessed 20 Nov 2018. The 8 episode-based measures are: elective outpatient percutaneous coronary intervention (PCI), knee arthroplasty, revascularization for lower extremity chronic critical limb ischemia, routine cataract removal with intraocular lens (IOL) implantation, screening surveillance colonoscopy, intracranial hemorrhage or cerebral infarction, simple pneumonia with hospitalization, and ST-elevation myocardial infarction (STEMI) with PCI.

3. Medicare Payment Advisory Commission. Report to Congress: Medicare Payment Policy. 2018. http://www.medpac.gov/docs/default-source/reports/mar18_medpac_entirereport_sec.pdf. Accessed 3 Jan 2019. 